Macroinvertebrate Communities and Habitat in Luxapalila Creek, Mississippi and Alabama

Barry S. Payne, Peter Smiley, and Andrew C. Miller

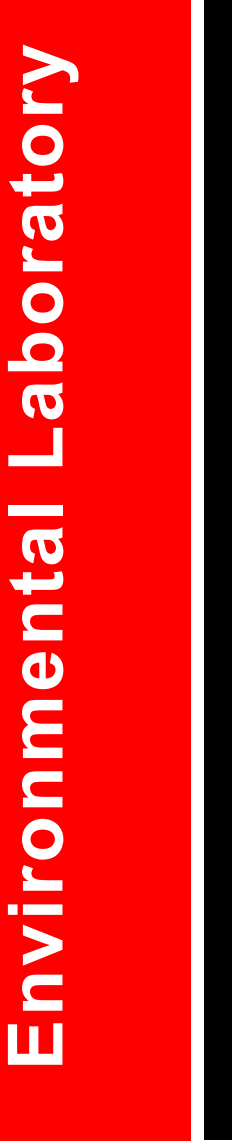


The contents of this report are not to be used for advertising, publication, or promotional purposes. Citation of trade names does not constitute an official endorsement or approval of the use of such commercial products.

The findings of this report are not to be construed as an official Department of the Army position, unless so designated by other authorized documents. 


\section{Macroinvertebrate Communities and Habitat in Luxapalila Creek, Mississippi and Alabama}

by Barry S. Payne, Andrew C. Miller

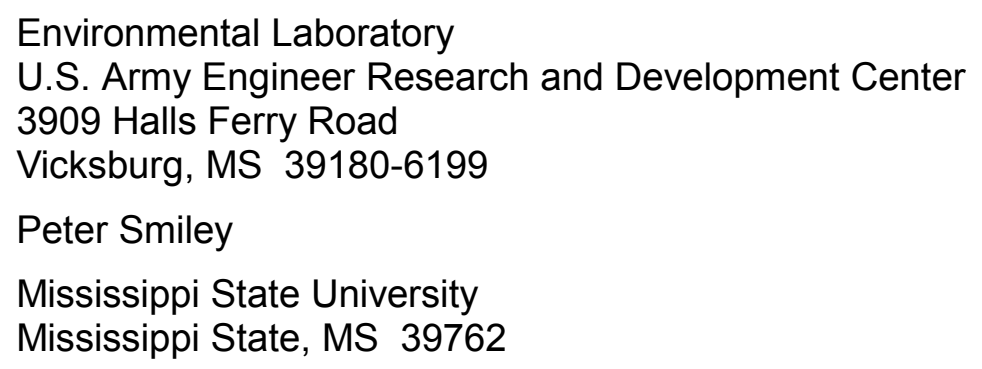

Final report

Approved for public release; distribution is unlimited 


\section{Contents}

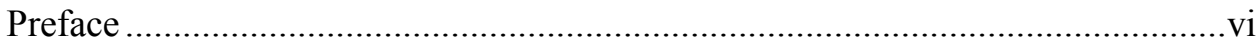

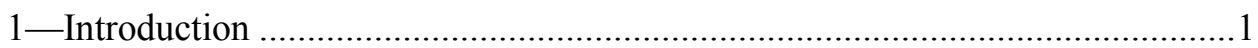

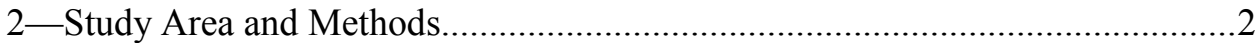

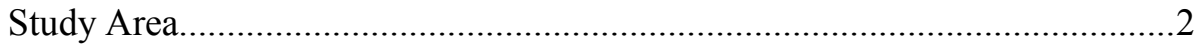

Site Selection and Data Collection ............................................................2

Before and After Project Comparisons.......................................................4

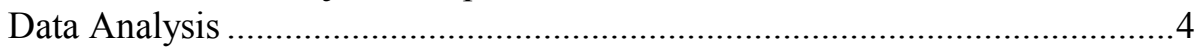

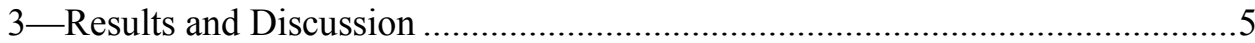

Habitat Characteristics ............................................................................

Macroinvertebrate Community Characteristics ........................................ 7

Before and After Project Comparisons..........................................................11

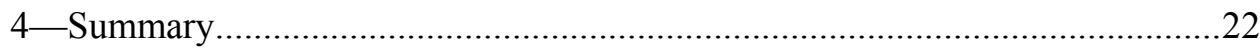

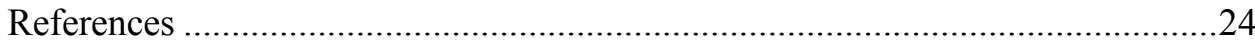

SF 298

\section{List of Figures}

Figure 1. Study sites of Luxapalila Creek, Mississippi and

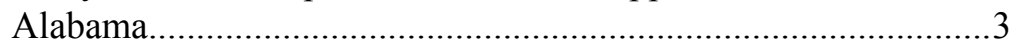

Figure 2. Relative abundance of functional feeding groups of macroinvertebrates from fall samples from pools in three channel types

Figure 3. Relative abundance of functional feeding groups of macroinvertebrates from spring samples from pools in three channel types 
Figure 4. Relative abundance of functional feeding groups of macroinvertebrates from fall samples from riffles in three channel types

Figure 5. Relative abundance of functional feeding groups of macroinvertebrates from spring samples from riffles in three channel types

\section{List of Tables}

Table 1. Summary of Mean Geomorphological and Vegetative Characteristics of Streambanks Within the Recently Channelized, Historically Channelized, and Unchannelized Portions of Luxapalila Creek

Table 2. Summary of Mean Habitat Parameters of Pools Among All Channel Types and Sampling Periods 6

Table 3. Summary of Mean Habitat Parameters of Riffles Among

All Channel Types and Sampling Periods

Table 4. Summary of Two Factor ANOVA Results and Factor Means of Pool Habitat Parameters That Exhibited Significant Single Factor Effects. 8

Table 5. Summary of Two Factor ANOVA Results and Factor Means of Riffle Habitat Parameters That Exhibited

Significant Single Factor Effects 9

Table 6. Relative Abundance of Macroinvertebrate Taxa From Pools in Three Channel Types and Two Sampling Periods

Table 7. Relative Abundance of Macroinvertebrate Functional Groups From Pools in Three Channel Types and Two Sampling Periods

Table 8. Relative Abundance of Macroinvertebrate Functional Groups From Riffles in Three Channel Types and Two Sampling Periods

Table 9. Summary of Two Factor ANOVA Results and Factor Means of the Density of the Five Most Common Macroinvertebrate Taxa in Pools That Exhibited Significant Single Factor Effects 
Table 10. Summary of Two Factor ANOVA Results and Factor Means of the Density of the Five Most Common Macroinvertebrate Taxa in Riffles That Exhibited Significant Single Factor Effects .16

Table 11. Mean Density of Macroinvertebrate Taxa From Pools in Three Channel Types and Two Sampling Periods

Table 12. Mean Density of Macroinvertebrate Taxa From Riffles in Three Channel Types and Two Sampling Periods......................19

Table 13. Relative Abundance of Macroinvertebrate Taxa From Pools in the Recently Altered Portion of Luxapalila Creek Among Pre- and Post-Alteration Sampling Periods and Two Seasons

Table 14. Relative Abundance of Macroinvertebrate Taxa From Riffles in the Recently Altered Portion of Luxapalila Creek Among Pre- and Post-Alteration Sampling Periods and Two Seasons 


\section{Preface}

The study reported herein was conducted by the U.S. Army Engineer Research and Development Center (ERDC), Vicksburg, MS, for the U.S. Army Engineer District, Mobile. The purpose was to analyze macroinvertebrate community composition and habitat at a series of pools and riffles in Luxapalila Creek in relation to recent construction of flood control measures in the lower portion of that stream.

Fieldwork was conducted by Mr. Peter Smiley, Mississippi State University (MSU), with the assistance of Mr. Will Green, University of Southern Mississippi. Drs. Andrew C. Miller, Environmental Laboratory (EL), ERDC, and Eric Dibble, MSU, provided guidance during conduct of this study.

During the conduct of this study, Dr. Edwin A. Theriot was Acting Director, EL; Dr. Dave Tazik was Chief, Ecosystem Evaluation and Engineering Division (EEED), EL; and Mr. Larry Sanders was Acting Chief, Aquatic Ecology and Invasive Species Branch (AEIB), EEED. Authors of this report were Drs. Barry S. Payne and Andrew C. Miller, AEIB, and Mr. Smiley.

At the time of publication of this report, Director of ERDC was Dr. James R. Houston. Commander and Executive Director was COL John W. Morris III, EN.

This report should be cited as follows:

Payne, B. S., Smiley, P., and Miller, A. C. (2001). "Macroinvertebrate communities and habitat in Luxapalila Creek, Mississippi and Alabama," ERDC/EL TR-01-34, U.S. Army Engineer Research and Development Center, Vicksburg, MS.

The contents of this report are not to be used for advertising, publication, or promotional purposes. Citation of trade names does not constitute an official endorsement or approval of the use of such commercial products. 


\section{Introduction}

The U.S. Army Engineer District, Mobile constructed flood control measures in the lower reach of Luxapalila Creek from 1998-2000. These measures included clearing and snagging, bank protection, excavation of pools, and construction of rock rubble weirs. The measures were designed to protect rural areas upstream of Columbus, MS, against floods expected to occur every 1.5 years, and to protect urban areas in Columbus from floods expected to occur every 5 years. Many aquatic habitats are altered by channel diversion, modification, or construction of dams (Standford and Ward 1979). Consequently, potentially adverse impacts to stream biota are a concern.

From 1987-1989, a study was conducted of stream characteristics and the macroinvertebrate community at a set of four riffles and four pools in an approximately 30-mile (48-km) reach of Luxapalila Creek extending from Columbus, MS, to Millport, AL (Payne et al. 1991; Payne and Miller 1991). The purpose of that study was to describe baseline conditions that could be subsequently compared to conditions revealed by a similar post-project study. This report presents the results of the post-project study of physical and biological conditions in Luxapalila Creek.

Emphasis in both studies was on macroinvertebrates, especially the more sessile taxa of fully aquatic organisms and aquatic life stages of insects. These animals are often used to monitor stream conditions and changes (Barbour et al. 1999). Density and diversity of these animals reflect geomorphologic, substratum, and water quality conditions. 


\section{Study Area and Methods}

\section{Study Area}

Luxapalila Creek watershed is situated within the northern edge of the Gulf Coastal Plains Physiographic Province. Luxapalila Creek is a tributary of the Tombigbee River and originates in Alabama and flows through northeast Mississippi (Payne et al. 1991). A 41.8-km section of Luxipalila Creek extending from Winfield, AL, to the Alabama-Mississippi state line was channelized in 1922, and then the lower $3.4 \mathrm{~km}$ was channelized in 1967 (Payne et al. 1991). In 1996, channelization and widening of the lower reaches near Columbus, MS, occurred.

Currently, three channel types are present within Luxapalila Creek (Figure 1). The downstream portion includes a recently channelized reach near Columbus, MS. The middle reach, near Steens, MS, has never been channelized. The very straight, upstream reach in Alabama was channelized approximately 80 years ago.

\section{Site Selection and Data Collection}

Two study sites were selected from within each of the different channel types and sampled as part of the present study (Figure 1). Sites 1 and 2 are located within the recently channelized portion and correspond with sites 1 and 2 studied by Payne et al. (1991). Site 1 is located downstream of Highway 182, and site 2 is located near the confluence of Magby Creek. Sites 3 and 4 are located within the unchannelized portion of Luxapalila Creek. Site 3 is located downstream of Mississippi Highway 12, and site 4 is located downstream of Gunshoot Road. Sites 5 and 6 are located within the historically channelized portion of Luxapalila Creek in Alabama. Site 5 is near Highway 17 in Millport, AL, while site 6 is downstream of Highway 49 near Kennedy, AL.

A previous study compared the macroinvertebrate communities of pools and riffles within Luxapalila Creek (Payne et al. 1991; Payne and Miller 1991). In the present study a space for time assessment was conducted to examine how macroinvertebrate communities and physical habitat within a microhabitat type varied among the three channel types and two seasonal periods (fall and spring). 


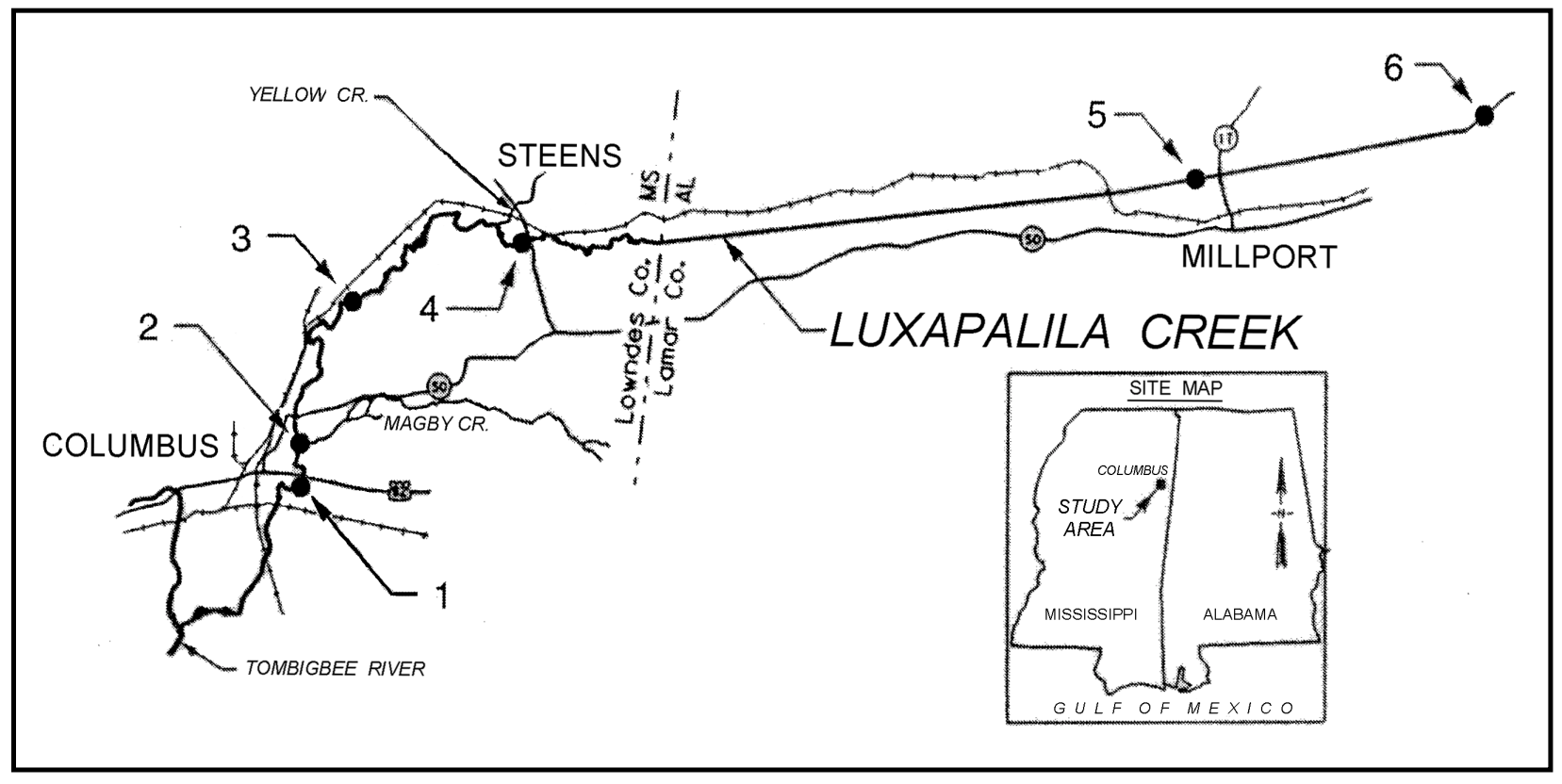

Figure 1. Study sites of Luxapalila Creek, Mississippi and Alabama

Preliminary observations indicated that distinct microhabitat types were present within the unchannelized and historically channelized portions of Luxapalila Creek. However, distinct pools and riffles were not present within the recently channelized portion of Luxapalila Creek. Pools and riffles within the recently channelized portion of Luxapalila Creek were delineated using qualitative habitat characteristics to define each microhabitat type to facilitate comparisons among channel types. Pools were considered to be areas of slow moving water greater than $0.56 \mathrm{~m}$ in depth with little surface turbulence, whereas riffles were defined as areas of fast moving water less than $0.56 \mathrm{~m}$ deep and exhibiting greater surface turbulence than pools.

Macroinvertebrates and physical habitat data were collected from each of the selected pools and riffles during four sampling periods: fall 1998, spring 1999, fall 1999, and spring 2000). Macroinvertebrates were sampled with a hand-held coring device (Miller and Bingham 1987) that sampled $0.0079 \mathrm{sq} \mathrm{m}$. Five cores for macroinvertebrate samples were taken within each microhabitat type (total cores per sampling period $=180$ ).

Water quality parameters ( $\mathrm{pH}$, temperature, dissolved oxygen, and turbidity) and estimated percent canopy cover in the middle of the sampling area of each microhabitat were measured during each sampling period. Depth, velocity, and substrate types were measured at three points along a transect located in the middle of the sampling area within each microhabitat type. Substrate types were visually classified in the field as either clay, sand, gravel, woody debris, or riprap.

Macroinvertebrate samples were preserved with 10-percent formalin solution in the field. In the laboratory, an elutriation process (Payne et al. 1991) was used to remove macroinvertebrates from the sediment. Macroinvertebrate samples were 
swirled in a $12-\mathrm{L}$ bucket and poured through a $250-\mu$ mesh sieve. Each sample was elutriated five times. Previous work indicated that this process removes 90 to 100 percent of macroinvertebrates within a sample (Payne et al. 1991). Macroinvertebrates were removed from elutriated samples with the aid of a stereomicroscope. Organisms were first sorted to major groups, such as chironomids, oligochaetes, and ephemeropterans, and counted.

Following the initial identifications, all sorted samples were recounted and further identifications were made. The following animals were only identified to major group (typically class): segmented worms (Oligochaetes), roundworms (Nematodes), horsehair worms (Nematomorpha), water mites (Hydracarina), planaria (Platyhelminthes), leeches (Hirudinea), amphipods (Amphipoda), crayfish (Decapoda), isopods (Isopoda), snails (Gastropoda), mussels (Bivalvia), springtails (Collembola), crickets (Orthoptera), and megalopterans (Megaloptera). The following taxa were identified to family level when possible: Coleoptera, Diptera, Ephemeroptera, Hemiptera, Odonata, Plecoptera, and Trichoptera. This level of taxonomic resolution allowed enumeration per functional feeding group.

\section{Before and After Project Comparisons}

Sites 1 and 2 (of the present study) were located within the recently channelized reach that was represented by sites 1,2 , and 3 of the previous study (Payne et al. 1991). Sites 5 and 6 (of the present study) fell in the historically channelized reach, as did site 4 of the previous study. At a level of river reach, comparisons were thus possible of pre- and post-project conditions in the recently channelized and historically channelized portions of the stream. At a finer level of spatial detail, riffles and pools from sites 1 and 2 of Payne et al. (1991) were within $200 \mathrm{~m}$ of sites 1 and 2 of the present study. Therefore, macroinvertebrate community structure at those two sites was specifically compared before and after project construction. Sampling methods and efforts with respect to macroinvertebrates were equal between pre- and post-alteration periods. In the 1981-1989 study, taxonomic resolution involved genus- and species-level identification of oligochaetes and dipterans to the maximum extent feasible. In the present study, identifications were made to the family level.

\section{Data Analysis}

Differences in mean physical habitat parameters among the different channel types and seasonal periods were assessed using a two factor analysis of variance (ANOVA) coupled with Student Newman-Keul's SNK multiple range test. Assumptions of ANOVA were tested prior to statistical testing, and if the data did not meet the assumptions, then they were transformed using $\log (\mathrm{x}+1)$ transformation. Variables calculated as percents (percent canopy cover, percent sand, etc.) were arcsine square root transformed prior to statistical testing (Zar 1987). Univariate statistical tests were conducted using SigmaStat 2.0 for Windows (Jandel Corporation 1995). Significance level for all univariate tests was $\mathrm{P}<0.05$. 


\section{Results and Discussion}

\section{Habitat Characteristics}

Geomorphological and vegetative characteristics of streambanks differed among recently channelized (RC), historically channelized (HC), and unchannelized (UC) portions of Luxapalila Creek (Table 1). Banks were steeper in the $\mathrm{HC}$ and UC than RC portions of the creek. The stream was narrowest in the UC portion. Trees were almost absent and shrubs were not common in the RC portion. Riprap was abundant in the RC but not the other portions. Bare soil comprised half of the streambank in the UC portion, one fifth in the RC portion, but one tenth in the $\mathrm{HC}$ portion of the creek. Canopy cover was absent in the $\mathrm{RC}$ portion of the creek, high to moderate in the HC portion, and moderate in the UC portion.

\begin{tabular}{|c|c|c|c|}
\hline \multicolumn{4}{|c|}{$\begin{array}{l}\text { Table } 1 \\
\text { Summary of Mean Geomorphological and Vegetative Characteristics } \\
\text { of Streambanks Within the Recently Channelized (RC), Historically } \\
\text { Channelized (HC), and the Unchannelized (UC) Portions of } \\
\text { Luxapalila Creek }\end{array}$} \\
\hline Characteristic & $\mathrm{RC}$ & $\mathrm{HC}$ & UC \\
\hline Bank angle, deg & 17.63 & 29.36 & 27.87 \\
\hline Bank height, $\mathrm{m}$ & 5.15 & 3.52 & 1.98 \\
\hline Top bank width, $\mathrm{m}$ & 57.54 & 42.06 & 26.93 \\
\hline Bottom channel width, $\mathrm{m}$ & 28.21 & 29.12 & 17.84 \\
\hline Percent trees (woody vegetation $>5 \mathrm{~m}$ ) & 0.03 & 5.35 & 6.25 \\
\hline Percent shrub (woody vegetation $<5 \mathrm{~m}$ ) & 11.71 & 34.38 & 22.50 \\
\hline Percent herbaceous vegetation & 39.05 & 49.72 & 23.82 \\
\hline Percent riprap & 27.89 & 2.99 & 0.42 \\
\hline Percent bare soil & 21.32 & 7.71 & 47.01 \\
\hline
\end{tabular}

Despite geomorphic and vegetative cover differences, water quality in pools (Table 2) and riffles (Table 3) was good and similar among all three portions of the creek. Measurements of $\mathrm{pH}$ indicated a very slightly acidic condition (6.7 to 6.8). Dissolved oxygen was high $(9.7$ to $10.4 \mathrm{mg} / \mathrm{L})$ at temperatures ranging from 12.5 to $15.7^{\circ} \mathrm{C}$. Turbidity was low (8.0 to $\left.11.5 \mathrm{NTU}\right)$. 


\begin{tabular}{|c|c|c|c|}
\hline \multicolumn{4}{|c|}{$\begin{array}{l}\text { Table } 2 \\
\text { Summary of Mean (Standard Error) Habitat Parameters of Pools } \\
\text { Among All Channel Types and Sampling Periods }\end{array}$} \\
\hline Habitat Parameter & RC & $\mathrm{HC}$ & UC \\
\hline \multicolumn{4}{|c|}{ Fall } \\
\hline $\mathrm{pH}$ & $6.77(0.20)$ & $6.84(0.20)$ & $6.74(0.17)$ \\
\hline Temperature, ${ }^{\circ} \mathrm{C}$ & $15.60(1.22)$ & $12.40(1.15)$ & $14.20(1.16)$ \\
\hline Dissolved oxygen, mg/L & $9.98(0.18)$ & $10.41(0.24)$ & $9.66(0.26)$ \\
\hline Turbidity, NTU & $8.44(2.95)$ & $8.04(2.95)$ & $11.46(4.19)$ \\
\hline Depth, m & $0.64(0.06)$ & $0.72(0.08)$ & $0.71(0.06)$ \\
\hline Velocity, m/sec & $0.37(0.13)$ & $0.08(0.05)$ & $0.00(0.00)$ \\
\hline Percent clay & $25.00(15.96)$ & $0.00(0.00)$ & $0.00(0.00)$ \\
\hline Percent sand & $0.00(0.00)$ & $83.33(9.62)$ & $25.00(15.96)$ \\
\hline Percent gravel & $66.67(13.61)$ & $16.67(9.62)$ & $75.00(15.96)$ \\
\hline Percent woody debris & $0.00(0.00)$ & $0.00(0.00)$ & $0.00(0.00)$ \\
\hline Percent riprap & $8.33(8.33)$ & $0.00(0.00)$ & $0.00(0.00)$ \\
\hline Percent canopy cover & $0.00(0.00)$ & $75.00(14.43)$ & $25.00(14.43)$ \\
\hline \multicolumn{4}{|c|}{ Spring } \\
\hline $\mathrm{pH}$ & $6.61(0.14)$ & $7.24(0.19)$ & $7.00(0.11)$ \\
\hline Temperature, ${ }^{\circ} \mathrm{C}$ & $23.48(0.36)$ & $22.23(0.43)$ & $23.33(0.25)$ \\
\hline Dissolved oxygen, mg/L & $8.20(0.16)$ & $8.35(0.28)$ & $7.52(0.13)$ \\
\hline Turbidity, NTU & $12.99(5.78)$ & $9.69(3.27)$ & $14.91(6.72)$ \\
\hline Depth, m & $0.68(0.06)$ & $0.89(0.04)$ & $0.72(0.03)$ \\
\hline Velocity, m/sec & $0.47(0.19)$ & $0.10(0.05)$ & $0.01(0.01)$ \\
\hline Percent clay & $0.00(0.00)$ & $0.00(0.00)$ & $0.00(0.00)$ \\
\hline Percent sand & $16.67(16.67)$ & $58.33(20.97)$ & $58.33(20.97)$ \\
\hline Percent gravel & $83.33(16.67)$ & $16.67(16.67)$ & $41.67(20.97)$ \\
\hline Percent woody debris & $0.00(0.00)$ & $25.00(8.00)$ & $0.00(0.00)$ \\
\hline Percent riprap & $0.00(0.00)$ & $0.00(0.00)$ & $0.00(0.00)$ \\
\hline Percent canopy cover & $0.00(0.00)$ & $87.50(12.50)$ & $25.00(14.43)$ \\
\hline
\end{tabular}

Pools ranged in depth from 0.64 to $0.72 \mathrm{~m}$ (Table 2). Velocity in the pools was very low in the HC and UC portions $(0.0$ to $0.1 \mathrm{~m} / \mathrm{sec})$ and moderate $(0.4 \mathrm{~m} / \mathrm{sec})$ in the RC portion. Riffles ranged only from 0.28 to $0.32 \mathrm{~m}$ deep (Table 3$)$; velocity was higher $(0.8 \mathrm{~m} / \mathrm{sec})$ in the UC than $\mathrm{HC}(0.5 \mathrm{~m} / \mathrm{sec})$ or RC $(0.4 \mathrm{~m} / \mathrm{sec})$ portions of the creek.

Substratum in pools differed markedly among the reaches (Table 2). Gravel (67 percent), hardpan clay ( 25 percent), and riprap ( 8 percent) were the only substratum types encountered in pools in the RC portion. In contrast, sand ( 83 percent) dominated the pools in the HC portion, while gravel ( 75 percent) was the predominant substratum in pools in the UC portion. Gravel was the predominant substratum (92 to 100 percent) of riffles in all stream reaches (Table 3).

ANOVA summaries of physical habitat and water quality characteristics of pools and riffles are provided in Tables 4 and 5, respectively. 


\begin{tabular}{|c|c|c|c|}
\hline \multicolumn{4}{|c|}{$\begin{array}{l}\text { Table } 3 \\
\text { Summary of Mean (Standard Error) Habitat Parameters of Riffles } \\
\text { Among All Channel Types and Sampling Periods }\end{array}$} \\
\hline Habitat Parameter & RC & $\mathrm{HC}$ & UC \\
\hline \multicolumn{4}{|c|}{ Fall } \\
\hline $\mathrm{pH}$ & $6.78(0.20)$ & $6.83(0.17)$ & $6.78(0.20)$ \\
\hline Temperature, ${ }^{\circ} \mathrm{C}$ & $15.70(1.32)$ & $12.45(1.14)$ & $14.18(1.14)$ \\
\hline Dissolved oxygen, mg/L & $9.87(0.22)$ & $10.16(0.19)$ & $9.57(0.30)$ \\
\hline Turbidity, NTU & $7.79(3.01)$ & $8.12(3.13)$ & $11.00(3.03)$ \\
\hline Depth, m & $0.28(0.04)$ & $0.25(0.06)$ & $0.32(0.03)$ \\
\hline Velocity, $\mathrm{m} / \mathrm{sec}$ & $0.36(0.14)$ & $0.45(0.09)$ & $0.79(0.04)$ \\
\hline Percent clay & $0.00(0.00)$ & $0.00(0.00)$ & $0.00(0.00)$ \\
\hline Percent sand & $0.00(0.00)$ & $0.08(0.08)$ & $0.00(0.00)$ \\
\hline Percent gravel & $100.00(0.00)$ & $91.67(8.33)$ & $100.00(0.00)$ \\
\hline Percent woody debris & $0.00(0.00)$ & $0.00(0.00)$ & $0.00(0.00)$ \\
\hline Percent riprap & $0.00(0.00)$ & $0.00(0.00)$ & $0.00(0.00)$ \\
\hline Percent canopy cover & $0.00(0.00)$ & $50.00(28.87)$ & $37.50(23.94)$ \\
\hline \multicolumn{4}{|c|}{ Spring } \\
\hline $\mathrm{pH}$ & $6.70(0.20)$ & $7.23(0.18)$ & $7.02(0.09)$ \\
\hline Temperature, ${ }^{\circ} \mathrm{C}$ & $23.58(0.51)$ & $22.18(0.39)$ & $23.40(0.25)$ \\
\hline Dissolved oxygen, mg/L & $8.13(0.20)$ & $8.51(0.26)$ & $7.78(0.18)$ \\
\hline Turbidity, NTU & $13.56(6.05)$ & $8.56(3.82)$ & $13.33(5.98)$ \\
\hline Depth, m & $0.45(0.04)$ & $0.24(0.05)$ & $0.52(0.04)$ \\
\hline Velocity, m/sec & $0.67(0.10)$ & $0.42(0.06)$ & $0.87(0.13)$ \\
\hline Percent clay & $0.00(0.00)$ & $0.00(0.00)$ & $0.00(0.00)$ \\
\hline Percent sand & $0.00(0.00)$ & $0.00(0.00)$ & $0.00(0.00)$ \\
\hline Percent gravel & $100.00(0.00)$ & $100.00(0.00)$ & $100.00(0.00)$ \\
\hline Percent woody debris & $0.00(0.00)$ & $0.00(0.00)$ & $0.00(0.00)$ \\
\hline Percent riprap & $0.00(0.00)$ & $0.00(0.00)$ & $0.00(0.00)$ \\
\hline Percent canopy cover & $0.00(0.00)$ & $50.00(28.87)$ & $37.50(23.94)$ \\
\hline
\end{tabular}

\section{Macroinvertebrate Community Characteristics}

A functional analysis of macroinvertebrate community composition indicated a remarkable degree of uniformity within the stream, regardless of season, reach, or habitat type within a reach (Tables 6-8; Figures 2-5). The macroinvertebrate community of Luxapalila Creek was consistently dominated by collector-gatherers and predators. This uniformity was true for recently channelized, historically channelized, and unchannelized stream reaches and for pools and riffles. Collector-gatherers comprised 55 to 83 percent of the fauna considering all locations and both seasons. Predatory macroinvertebrates comprised 15 to 45 percent of the fauna for both seasons. Shredders and scrapers comprised only 0 to 4 percent of the fauna, probably reflecting the lack of large gravel and cobble in the streambed for both seasons. Collector-filterers comprised from 0 to 18 percent of the fauna and were consistently least abundant in the historically channelized 


\begin{tabular}{|l}
\hline Table 4 \\
Summary of Two Factor ANOVA Results and Factor Means \\
(Standard Error) of Pool Habitat Parameters That Exhibited
\end{tabular}

upper reach of the stream (Tables 7 and 8). Collector-filterers were mostly the Asian clam, Corbicula fluminea, and a few native unionid mussels. These bivalves were enormously greater in size than the numerically dominant chironomids, oligochaetes, and similarly small organisms. Thus, the ecological significance of filtering to stream trophic dynamics almost certainly was greater than indicated by their numerical relative abundance.

Total density of four of the five most common macroinvertebrates in pools (midges, unsegmented worms, segmented worms, and mites) did not vary significantly among recently channelized, historically channelized, and 


\begin{tabular}{|c|c|c|c|c|c|}
\hline \multicolumn{6}{|c|}{$\begin{array}{l}\text { Table } 5 \\
\text { Summary of Two Factor ANOVA Results and Factor Means } \\
\text { (Standard Error) of Riffle Habitat Parameters That Exhibited } \\
\text { Significant Single Factor Effects }\end{array}$} \\
\hline \multirow[b]{2}{*}{ Channel type } & \multirow{2}{*}{$\begin{array}{l}\text { ANOVA } \\
\text { Results }\end{array}$} & \multicolumn{4}{|c|}{ Factor Means } \\
\hline & & RC & \multicolumn{2}{|l|}{$\mathrm{HC}$} & UC \\
\hline $\mathrm{pH}$ & NS & - & \multicolumn{2}{|l|}{-} & - \\
\hline Temperature, ${ }^{\circ} \mathrm{C}$ & NS & - & \multicolumn{2}{|l|}{-} & - \\
\hline Dissolved oxygen, mg/L & $S$ & $9.00(0.36) a b$ & \multicolumn{2}{|c|}{$9.34(0.35) \mathrm{a}$} & $8.68(0.37) b$ \\
\hline Turbidity, NTU & NS & - & \multicolumn{2}{|l|}{-} & - \\
\hline Depth, m & S & $0.36(0.04) a$ & \multicolumn{2}{|c|}{$0.24(0.04) b$} & $0.42(0.05) \mathrm{a}$ \\
\hline Velocity, $\mathrm{m} / \mathrm{sec}$ & $S$ & $0.51(0.10) \mathrm{a}$ & \multicolumn{2}{|c|}{$0.43(0.05) a$} & $0.83(0.06) b$ \\
\hline Percent clay & NS & - & \multicolumn{2}{|c|}{-} & - \\
\hline Percent sand & NS & - & \multicolumn{2}{|l|}{-} & - \\
\hline Percent gravel & NS & - & \multicolumn{2}{|l|}{-} & - \\
\hline Percent woody debris & NS & - & \multicolumn{2}{|l|}{-} & - \\
\hline Percent riprap & NS & - & \multicolumn{2}{|l|}{-} & - \\
\hline \multirow[t]{2}{*}{ Percent canopy cover } & NS & - & \multicolumn{2}{|l|}{-} & - \\
\hline & \multirow{2}{*}{$\begin{array}{l}\text { ANOVA } \\
\text { Results }\end{array}$} & \multicolumn{4}{|c|}{ Factor Means } \\
\hline Season & & Fall & \multicolumn{3}{|c|}{\begin{tabular}{l|l} 
& Spring \\
\end{tabular}} \\
\hline $\mathrm{pH}$ & NS & \multicolumn{2}{|r|}{ - } & \multicolumn{2}{|c|}{ Spring } \\
\hline Temperature, ${ }^{\circ} \mathrm{C}$ & $S$ & \multicolumn{2}{|l|}{$14.11(0.74)$} & \multicolumn{2}{|c|}{$23.05(0.28)$} \\
\hline Dissolved oxygen, mg/L & $\mathrm{S}$ & \multicolumn{2}{|l|}{$9.87(0.15)$} & \multicolumn{2}{|c|}{$8.14(0.14)$} \\
\hline Turbidity, NTU & NS & \multicolumn{2}{|l|}{-} & \multicolumn{2}{|c|}{-} \\
\hline Depth, m & $S$ & $0.28(0.03)$ & & 0.40 & .04) \\
\hline Velocity, $\mathrm{m} / \mathrm{sec}$ & NS & - & - & 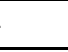 & \\
\hline Percent clay & NS & - & - & . & \\
\hline Percent sand & NS & - & - & . & \\
\hline Percent gravel & NS & - & - & . & \\
\hline Percent woody debris & NS & - & - & 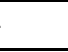 & \\
\hline Percent riprap & NS & - & - & - & \\
\hline Percent canopy cover & NS & - & - & & \\
\hline
\end{tabular}

unchannelized sections of the creek (Table 9). In contrast, bivalves (mostly $C$. fluminea) were much more abundant in recently channelized than historically channelized sections. Density was intermediate in the unchannelized portion of the creek. This pattern follows an upstream to downstream increase in C. fluminea density that might be expected due to trophic considerations, rather than channel modifications, applicable to a stream this size.

Seasonal differences in density of the most abundant taxa in pools were more evident than habitat differences (Table 9). Chironomid abundance in fall was more than twice that observed in spring. Oligochaetes were nearly four times more abundant in fall than spring. Bivalves were approximately 50 times more 


\begin{tabular}{|c|c|c|c|c|c|c|c|}
\hline \multicolumn{8}{|c|}{$\begin{array}{l}\text { Table } 6 \\
\text { Relative Abundance (Percent) of Macroinvertebrate Taxa From Pools } \\
\text { in Three Channel Types and Two Sampling Periods }\end{array}$} \\
\hline \multirow[b]{2}{*}{ Order } & \multirow[b]{2}{*}{ Family } & \multicolumn{3}{|c|}{ Fall } & \multicolumn{3}{|c|}{ Spring } \\
\hline & & RC & $\mathrm{HC}$ & UC & RC & $\mathrm{HC}$ & UC \\
\hline Bivalvia & & 6.768 & 0.464 & 1.474 & 0.205 & 0.211 & 0.124 \\
\hline \multirow[t]{6}{*}{ Coleoptera } & Dryopidae & 0.000 & 0.000 & 0.200 & 0.000 & 0.000 & 0.000 \\
\hline & Dytiscidae & 0.000 & 0.000 & 0.050 & 0.000 & 0.000 & 0.000 \\
\hline & Elmidae & 0.400 & 1.404 & 0.914 & 0.328 & 0.136 & 1.068 \\
\hline & \begin{tabular}{|l|} 
Gyrinidae \\
\end{tabular} & 0.000 & 0.000 & 0.000 & 0.000 & 0.000 & 0.000 \\
\hline & Staphylinidae & 0.000 & 0.000 & 0.050 & 0.000 & 0.000 & 0.000 \\
\hline & Unknown & 0.000 & 0.000 & 0.000 & 0.000 & 0.093 & 0.000 \\
\hline Collembola & & 0.054 & 0.155 & 0.000 & 0.109 & 0.093 & 0.000 \\
\hline \multirow[t]{9}{*}{ Diptera } & Ceratapogonidae & 0.749 & 5.032 & 3.445 & 1.218 & 2.270 & 2.009 \\
\hline & \begin{tabular}{|l|} 
Chironomidae \\
\end{tabular} & 53.382 & 59.990 & 65.658 & 69.097 & 67.269 & 47.313 \\
\hline & Empididae & 0.098 & 0.108 & 0.249 & 0.000 & 0.085 & 0.281 \\
\hline & Nymphomyiidae & 0.000 & 0.000 & 0.000 & 0.000 & 0.291 & 0.000 \\
\hline & Simuliidae & 0.000 & 0.000 & 0.000 & 1.040 & 0.366 & 0.157 \\
\hline & Tabanidae & 0.000 & 0.000 & 0.000 & 0.000 & 0.000 & 0.048 \\
\hline & \begin{tabular}{|l|} 
Tanyderidae \\
\end{tabular} & 0.000 & 0.000 & 0.000 & 0.000 & 0.000 & 0.000 \\
\hline & Tipulidae & 0.000 & 0.000 & 0.000 & 0.000 & 0.000 & 0.000 \\
\hline & Unknown & 0.366 & 0.000 & 0.100 & 0.041 & 0.117 & 0.051 \\
\hline \multirow[t]{10}{*}{ Ephemeroptera } & Baetidae & 0.000 & 0.000 & 0.000 & 0.000 & 0.175 & 0.096 \\
\hline & \begin{tabular}{|l|} 
Baetiscidae \\
\end{tabular} & 0.000 & 0.000 & 0.125 & 0.000 & 0.000 & 0.000 \\
\hline & Caenidae & 0.000 & 0.000 & 0.000 & 0.547 & 0.000 & 0.102 \\
\hline & Ephemeridae & 0.161 & 0.668 & 0.226 & 0.000 & 0.210 & 0.000 \\
\hline & Heptageniidae & 1.020 & 0.077 & 0.449 & 0.323 & 0.000 & 0.000 \\
\hline & Isonychiidae & 0.161 & 0.000 & 0.000 & 0.000 & 0.000 & 0.000 \\
\hline & Polmitarcyidae & 0.000 & 0.000 & 0.000 & 0.000 & 0.000 & 0.000 \\
\hline & Potamanthidae & 0.000 & 0.000 & 0.000 & 0.164 & 0.093 & 0.000 \\
\hline & \begin{tabular}{|l|} 
Tricorythidae \\
\end{tabular} & 0.000 & 0.077 & 0.000 & 0.657 & 0.000 & 0.191 \\
\hline & Unknown & 1.174 & 1.558 & 0.100 & 3.255 & 1.107 & 0.673 \\
\hline Gastropoda & & 2.631 & 0.185 & 0.848 & 0.123 & 0.000 & 0.000 \\
\hline Hirudinea & & 0.166 & 0.000 & 0.000 & 0.000 & 0.000 & 0.000 \\
\hline Hydracarina & & 2.687 & 4.504 & 0.984 & 1.133 & 3.908 & 1.372 \\
\hline Isopoda & & 0.000 & 0.000 & 0.000 & 0.000 & 0.155 & 0.000 \\
\hline Megaloptera & & 0.000 & 0.000 & 0.000 & 0.000 & 0.233 & 0.000 \\
\hline Nematoda & & 14.136 & 13.908 & 18.853 & 12.417 & 15.154 & 40.410 \\
\hline Nematomorpha & & 0.000 & 0.000 & 0.050 & 0.000 & 0.000 & 0.000 \\
\hline \multirow[t]{4}{*}{ Odonata } & Coenagrionidae & 0.128 & 0.000 & 0.000 & 0.000 & 0.000 & 0.153 \\
\hline & Corduliidae & 0.000 & 0.309 & 0.000 & 0.000 & 0.000 & 0.000 \\
\hline & Gomphidae & 0.054 & 0.103 & 0.266 & 0.000 & 0.093 & 0.000 \\
\hline & Unknown & 0.036 & 0.077 & 0.116 & 0.041 & 0.000 & 0.000 \\
\hline Oligochaeta & & 13.601 & 7.307 & 4.288 & 8.141 & 6.921 & 5.078 \\
\hline Orthoptera & Tridactylidae & 0.000 & 0.000 & 0.025 & 0.000 & 0.233 & 0.000 \\
\hline \multirow[t]{3}{*}{ Plecoptera } & Perlidae & 0.357 & 0.155 & 0.000 & 0.370 & 0.000 & 0.000 \\
\hline & Perlodidae & 0.000 & 0.000 & 0.050 & 0.312 & 0.214 & 0.000 \\
\hline & Unknown & 0.000 & 0.116 & 0.000 & 0.000 & 0.133 & 0.000 \\
\hline \multirow[t]{8}{*}{ Trichoptera } & \begin{tabular}{|l|} 
Brachycentridae \\
\end{tabular} & 0.000 & 0.000 & 0.000 & 0.000 & 0.000 & 0.096 \\
\hline & Hydropsychidae & 0.268 & 0.000 & 0.000 & 0.000 & 0.000 & 0.000 \\
\hline & Hydroptilidae & 0.514 & 0.147 & 0.299 & 0.000 & 0.000 & 0.191 \\
\hline & Leptoceridae & 0.612 & 3.619 & 0.831 & 0.000 & 0.000 & 0.281 \\
\hline & Philopotamidae & 0.223 & 0.000 & 0.050 & 0.066 & 0.000 & 0.000 \\
\hline & Polycentropodidae & 0.027 & 0.000 & 0.100 & 0.000 & 0.000 & 0.000 \\
\hline & Psychomyiidae & 0.000 & 0.000 & 0.000 & 0.000 & 0.058 & 0.000 \\
\hline & Unknown & 0.232 & 0.039 & 0.200 & 0.411 & 0.383 & 0.306 \\
\hline
\end{tabular}




\begin{tabular}{|c|c|c|c|c|c|c|}
\hline \multicolumn{7}{|c|}{$\begin{array}{l}\text { Table } 7 \\
\text { Relative Abundance (Percent) of Macroinvertebrate Functional } \\
\text { Groups From Pools in Three Channel Types and Two Sampling } \\
\text { Periods }\end{array}$} \\
\hline \multirow[b]{2}{*}{ Invertebrate Group } & \multicolumn{3}{|c|}{\begin{tabular}{|c|} 
Fall \\
\end{tabular}} & \multicolumn{3}{|c|}{ Spring } \\
\hline & RC & HC & UC & RC & HC & UC \\
\hline Collector-filterer & 7.49 & 0.46 & 1.63 & 1.48 & 0.67 & 0.38 \\
\hline Collector-gatherer & 69.80 & 74.81 & 72.36 & 82.51 & 76.51 & 55.00 \\
\hline Scraper & \begin{tabular}{|l|}
3.67 \\
\end{tabular} & 0.26 & 1.30 & 0.45 & 0.29 & 0.00 \\
\hline Shredder & 0.00 & 0.00 & 0.23 & 0.00 & 0.23 & 0.00 \\
\hline Predator & 18.52 & 24.32 & 24.19 & 15.56 & 22.29 & 44.43 \\
\hline Piercer & 0.52 & 0.15 & 0.30 & 0.00 & 0.00 & 0.19 \\
\hline
\end{tabular}

\begin{tabular}{|c|c|c|c|c|c|c|}
\hline \multicolumn{7}{|c|}{$\begin{array}{l}\text { Table } 8 \\
\text { Relative Abundance (Percent) of Macroinvertebrate Functional } \\
\text { Groups From Riffles in Three Channel Types and Two Sampling } \\
\text { Periods }\end{array}$} \\
\hline \multirow[b]{2}{*}{ Invertebrate Group } & \multicolumn{3}{|c|}{\begin{tabular}{|c|} 
Fall \\
\end{tabular}} & \multicolumn{3}{|c|}{ Spring } \\
\hline & $\mathrm{RC}$ & HC & UC & $\mathrm{RC}$ & $\mathrm{HC}$ & UC \\
\hline Collector-filterer & 3.94 & 2.11 & 6.39 & 1.21 & 0.67 & 18.18 \\
\hline Collector-gatherer & 71.73 & 79.80 & 62.07 & 83.03 & 81.93 & 63.32 \\
\hline Scraper & 4.18 & 0.63 & 2.12 & 0.80 & 0.00 & 0.78 \\
\hline Shredder & 0.00 & 0.19 & 0.00 & 0.00 & 0.21 & 0.00 \\
\hline Predator & 19.41 & 16.95 & 27.65 & 14.90 & 17.06 & 17.55 \\
\hline Piercer & 0.74 & 0.32 & \begin{tabular}{|l|}
1.77 \\
\end{tabular} & 0.06 & \begin{tabular}{|l|}
0.14 \\
\end{tabular} & \begin{tabular}{|l|}
0.17 \\
\end{tabular} \\
\hline
\end{tabular}

abundant in fall than spring. However, nematodes and mites did not show such seasonal differences.

In riffles, total density of chironomids, oligochaetes, and nematodes varied significantly among recently channelized, historically channelized, and unchannelized portions of the stream (Table 10). All taxa were highest in density in the recently channelized reach and least dense in the historically channelized reach. Density tended to be intermediate in the unchannelized reach. As with $C$.

fluminea, this patterns follows an upstream to downstream increase in density. Seasonal difference in macroinvertebrate density in riffles was similar to that seen in pools. Chironomids, oligochaetes, and nematodes were all denser in fall than spring (Table 10).

\section{Before and After Project Comparisons}

At the level of river reach, total macroinvertebrate density, dominated by chironomids, was substantially greater in the present study (Tables 11 and 12) than in the 1987-1989 study (Figure 3 in Payne and Miller 1991). In the previous study, a pool in the historically channelized reach supported 6,000 to 


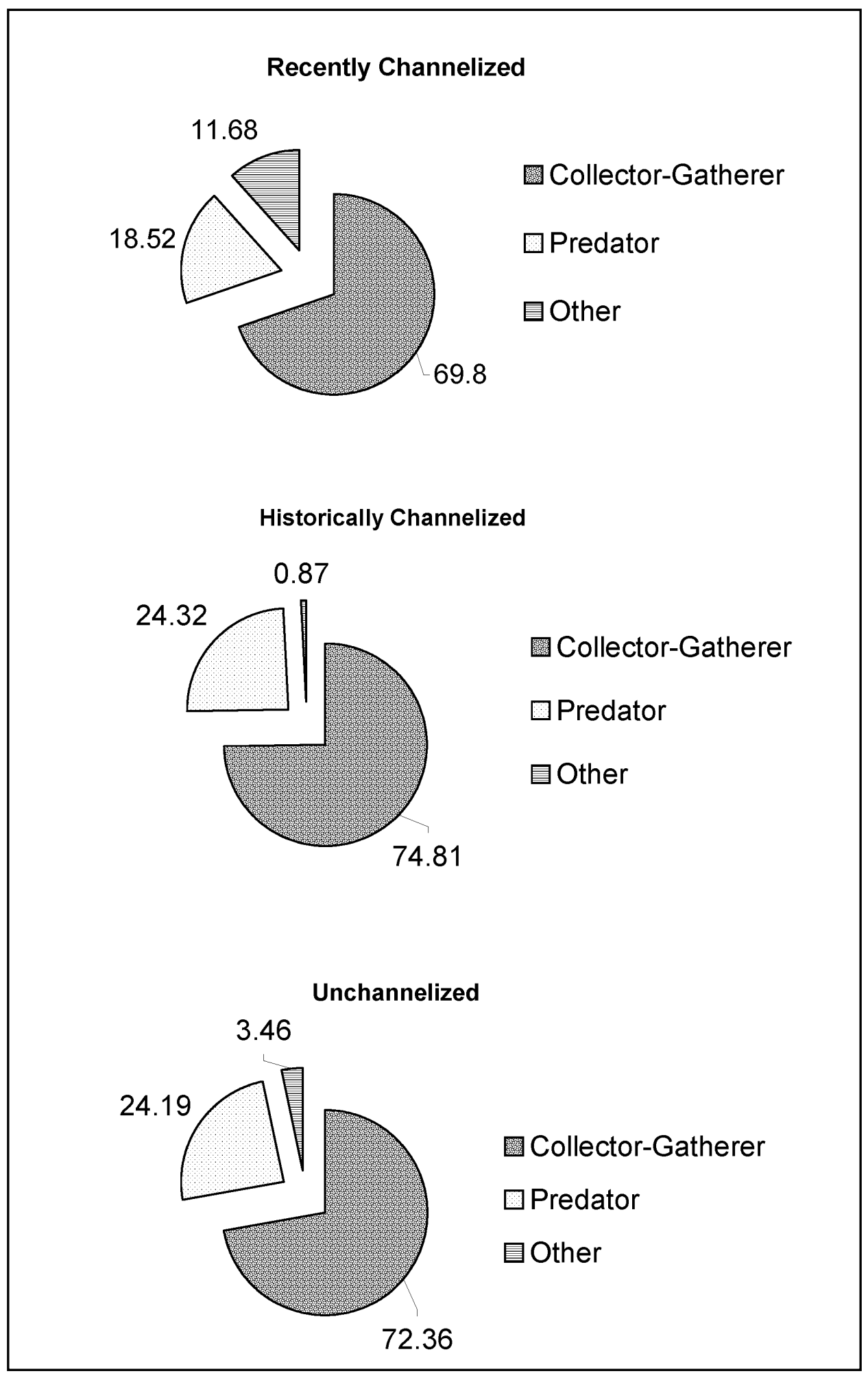

Figure 2. Relative abundance of functional feeding groups of macroinvertebrates from fall samples from pools in three channel types 


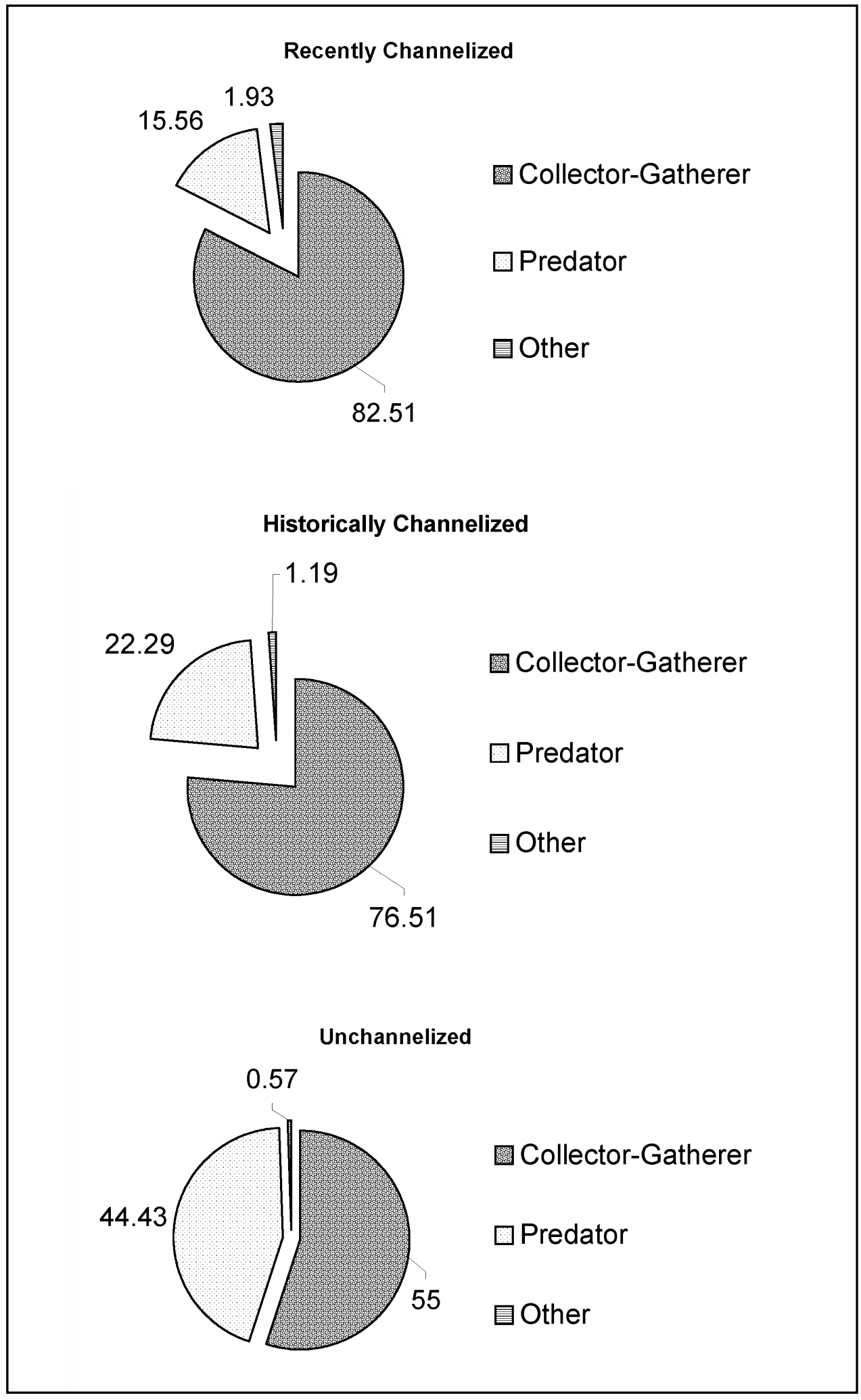

Figure 3. Relative abundance of functional feeding groups of macroinvertebrates from spring samples from pools in three channel types 


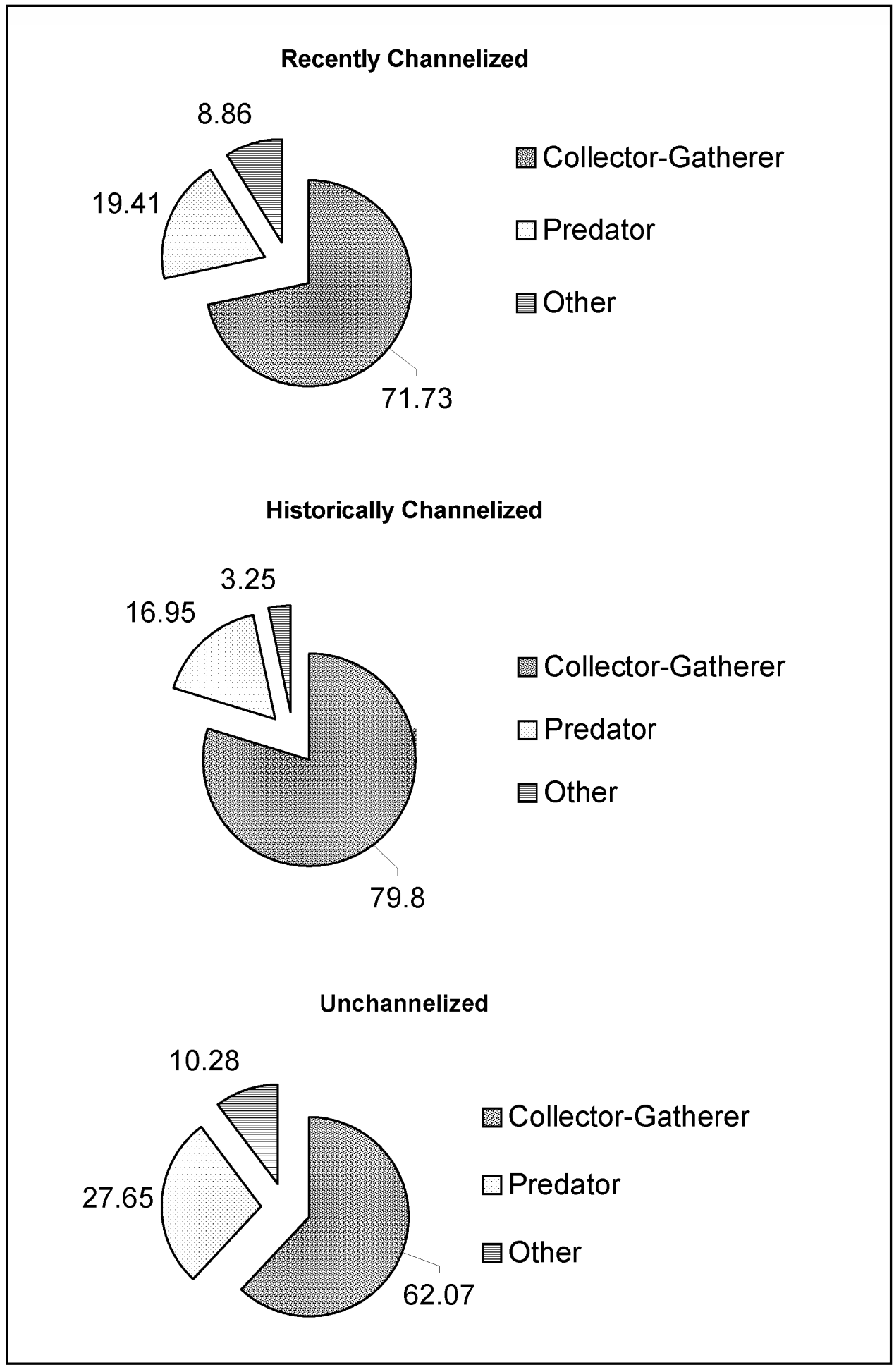

Figure 4. Relative abundance of functional feeding groups of macroinvertebrates from fall samples from riffles in three channel types 


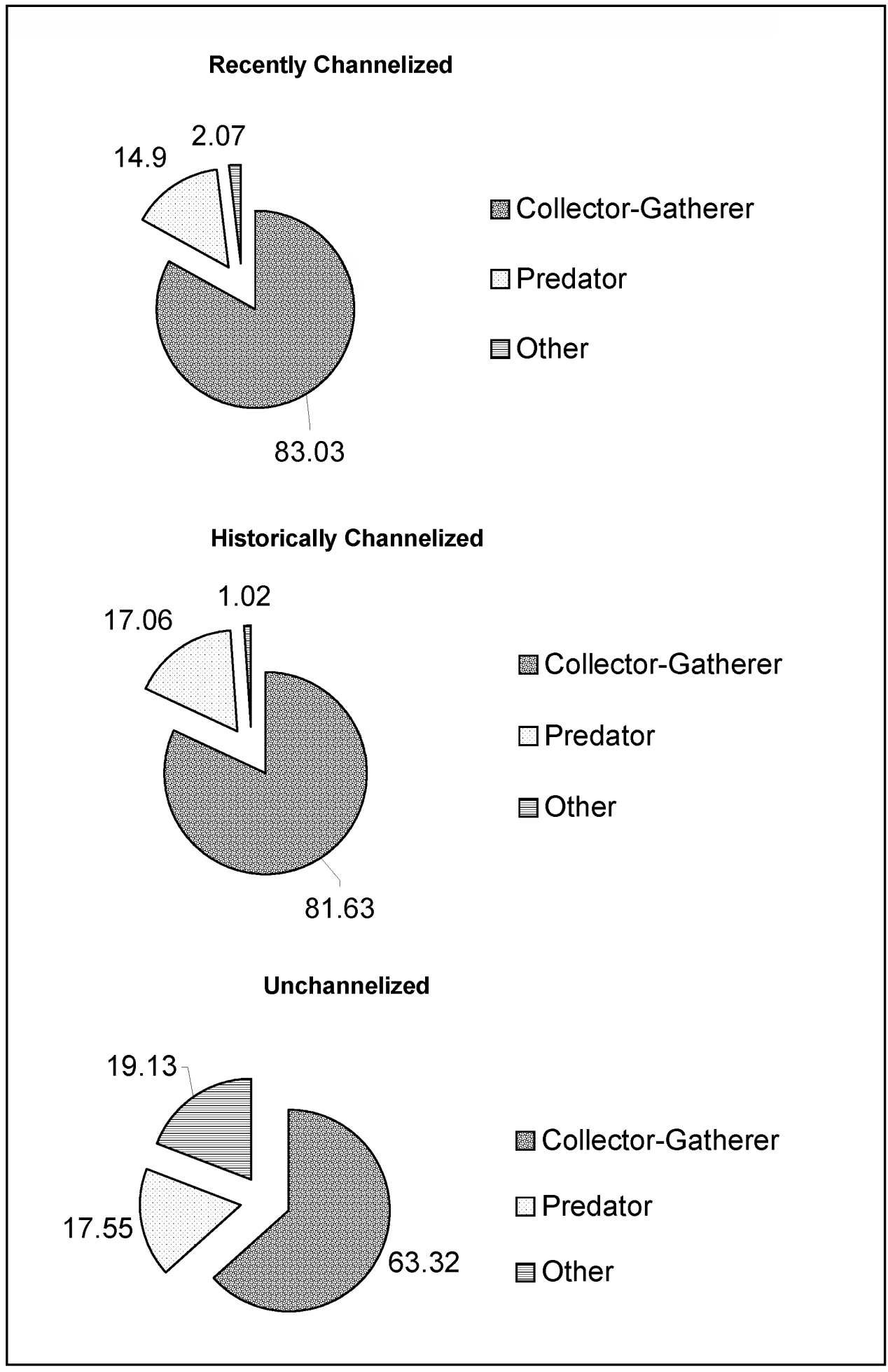

Figure 5. Relative abundance of functional feeding groups of macroinvertebrates from spring samples from riffles in three channel types 


\begin{tabular}{|l}
\hline Table 9 \\
Summary of Two Factor ANOVA Results and Factor Means \\
(Standard Error) of the Density of the Five Most Common \\
Macroinvertebrate Taxa in Pools That Exhibited Significant Single \\
Factor Effects
\end{tabular}

\begin{tabular}{|l}
\hline Table 10 \\
Summary of Two Factor ANOVA Results and Factor Means \\
(Standard Error) of the Density of the Five Most Common \\
Macroinvertebrate Taxa in Riffles That Exhibited Significant Single \\
Factor Effects
\end{tabular}




\begin{tabular}{|c|c|c|c|c|c|c|c|}
\hline \multicolumn{8}{|c|}{$\begin{array}{l}\text { Table } 11 \\
\text { Mean Density (Individuals per Square Meter) of Macroinvertebrate Taxa From Pools in } \\
\text { Three Channel Types and Two Sampling Periods }\end{array}$} \\
\hline \multirow[b]{2}{*}{ Order } & \multirow[b]{2}{*}{ Family } & \multicolumn{3}{|c|}{ Fall } & \multicolumn{3}{|c|}{$\begin{array}{l}\text { Spring } \\
\end{array}$} \\
\hline & & RC & $\mathrm{HC}$ & UC & RC & $\mathrm{HC}$ & UC \\
\hline Bivalvia & & 3202.25 & 151.90 & 374.12 & 31.65 & 22.91 & 16.46 \\
\hline \multirow[t]{6}{*}{ Coleoptera } & Dryopidae & 0.00 & 0.00 & 50.63 & 0.00 & 0.00 & 0.00 \\
\hline & Dytiscidae & 0.00 & 0.00 & 12.66 & 0.00 & 0.00 & 0.00 \\
\hline & Elmidae & 189.03 & 459.92 & 232.07 & 50.63 & 14.77 & 141.29 \\
\hline & Gyrinidae & 0.00 & 0.00 & 0.00 & 0.00 & 0.00 & 0.00 \\
\hline & Staphylinidae & 0.00 & 0.00 & 12.66 & 0.00 & 0.00 & 0.00 \\
\hline & Unknown & 0.00 & 0.00 & 0.00 & 0.00 & 10.13 & 0.00 \\
\hline Collembola & & 25.32 & 50.63 & 0.00 & 16.88 & 10.13 & 0.00 \\
\hline \multirow[t]{9}{*}{ Diptera } & Ceratapogonidae & 354.29 & 1648.82 & 874.40 & 187.76 & 246.61 & 265.82 \\
\hline & Chironomidae & 25257.38 & 19656.54 & 16662.87 & 10650.21 & 7309.03 & 6260.28 \\
\hline & Empididae & 46.41 & 35.44 & $\begin{array}{l}63.29 \\
\end{array}$ & 0.00 & 9.21 & 37.13 \\
\hline & Nymphomyiidae & 0.00 & 0.00 & 0.00 & 0.00 & 31.65 & 0.00 \\
\hline & Simuliidae & 0.00 & 0.00 & 0.00 & 160.34 & 39.78 & 20.80 \\
\hline & Tabanidae & 0.00 & 0.00 & 0.00 & 0.00 & 0.00 & 6.33 \\
\hline & Tanyderidae & 0.00 & 0.00 & 0.00 & 0.00 & 0.00 & 0.00 \\
\hline & Tipulidae & 0.00 & 0.00 & 0.00 & 0.00 & 0.00 & 0.00 \\
\hline & Unknown & 173.00 & 0.00 & 25.32 & 6.33 & 12.66 & 6.75 \\
\hline \multirow[t]{10}{*}{ Ephemeroptera } & Baetidae & 0.00 & 0.00 & 0.00 & 0.00 & 18.99 & 12.66 \\
\hline & Baetiscidae & 0.00 & 0.00 & 31.65 & 0.00 & 0.00 & 0.00 \\
\hline & Caenidae & 0.00 & 0.00 & 0.00 & 84.39 & 0.00 & 13.50 \\
\hline & \begin{tabular}{|l} 
Ephemeridae \\
\end{tabular} & 75.95 & 218.81 & 57.38 & 0.00 & 22.78 & 0.00 \\
\hline & Heptageniidae & 482.42 & 25.32 & 113.92 & 49.79 & 0.00 & 0.00 \\
\hline & Isonychiidae & 75.95 & 0.00 & 0.00 & 0.00 & 0.00 & 0.00 \\
\hline & Polmitarcyidae & 0.00 & 0.00 & 0.00 & 0.00 & 0.00 & 0.00 \\
\hline & Potamanthidae & 0.00 & 0.00 & 0.00 & 25.32 & 10.13 & 0.00 \\
\hline & Tricorythidae & 0.00 & 25.32 & 0.00 & 101.27 & 0.00 & 25.32 \\
\hline & Unknown & 555.56 & 510.55 & 25.32 & 501.69 & 120.25 & 89.03 \\
\hline Gastropoda & & 1244.73 & 60.76 & 215.19 & 18.99 & 0.00 & 0.00 \\
\hline Hirudinea & & 78.76 & 0.00 & 0.00 & 0.00 & 0.00 & 0.00 \\
\hline Hydracarina & & 1271.31 & 1475.77 & 249.65 & 174.68 & 424.64 & 181.56 \\
\hline Isopoda & & 0.00 & 0.00 & 0.00 & 0.00 & 16.88 & 0.00 \\
\hline Megaloptera & & 0.00 & 0.00 & 0.00 & 0.00 & 25.32 & 0.00 \\
\hline Nematoda & & 6688.47 & 4557.08 & 4784.67 & 1913.92 & 1646.51 & 5346.90 \\
\hline Nematomorpha & & 0.00 & 0.00 & 12.66 & 0.00 & 0.00 & 0.00 \\
\hline \multirow[t]{4}{*}{ Odonata } & Coenagrionidae & 60.34 & 0.00 & 0.00 & 0.00 & 0.00 & 20.25 \\
\hline & Corduliidae & 0.00 & 101.27 & 0.00 & 0.00 & 0.00 & 0.00 \\
\hline & Gomphidae & 25.32 & 33.76 & 67.51 & 0.00 & 10.13 & 0.00 \\
\hline & Unknown & 16.88 & 25.32 & 29.54 & 6.33 & 0.00 & 0.00 \\
\hline Oligochaeta & & 6435.16 & 2394.09 & 1088.19 & 1254.85 & 752.00 & 671.91 \\
\hline Orthoptera & Tridactylidae & 0.00 & 0.00 & 6.33 & 0.00 & 25.32 & 0.00 \\
\hline \multirow[t]{3}{*}{ Plecoptera } & Perlidae & 168.78 & 50.63 & 0.00 & 56.96 & 0.00 & 0.00 \\
\hline & Perlodidae & 0.00 & 0.00 & 12.66 & 48.10 & 23.21 & 0.00 \\
\hline & Unknown & 0.00 & 37.97 & 0.00 & 0.00 & 14.47 & 0.00 \\
\hline \multirow[t]{8}{*}{ Trichoptera } & Brachycentridae & 0.00 & 0.00 & 0.00 & 0.00 & 0.00 & 12.66 \\
\hline & Hydropsychidae & 126.58 & 0.00 & 0.00 & 0.00 & 0.00 & 0.00 \\
\hline & Hydroptilidae & 243.32 & 48.10 & 75.95 & 0.00 & 0.00 & 25.32 \\
\hline & Leptoceridae & 289.73 & 1185.90 & 210.97 & 0.00 & 0.00 & 37.13 \\
\hline & Philopotamidae & 105.49 & 0.00 & 12.66 & 10.13 & 0.00 & 0.00 \\
\hline & Polycentropodidae & 12.66 & 0.00 & 25.32 & 0.00 & 0.00 & 0.00 \\
\hline & Psychomyiidae & 0.00 & 0.00 & 0.00 & 0.00 & 6.33 & 0.00 \\
\hline & Unknown & 109.70 & 12.66 & 50.63 & 63.29 & 41.59 & 40.51 \\
\hline
\end{tabular}


8,000 macroinvertebrates per square meter. In contrast, pools in the historically channelized reach during the present study yielded density estimates of 10,000 to 30,000 individuals per square meter (Table 11). In riffles, density estimates were 2,000 to 12,000 individuals per square meter in $1987-1989$ versus 8,000 to 31,000 individuals per square meter (Table 12) in the present study.

The detailed spatial comparison in the recently channelized reach, where two particular pool and riffle pairs were sampled in both the present (post-project) and previous (pre-project) study, did not indicate adverse effects on the macroinvertebrate community. Once again, macroinvertebrate density was substantially greater in the present than previous study. Focusing on fall data, when density was greatest, macroinvertebrate density in riffles averaged 68,000 versus 28,000 individuals per square meter in the present and previous study, respectively. In pools, density of macroinvertebrates in fall equaled 31,000 and 10,000 individuals per square meter in the present and previous studies, respectively. Despite the overall differences in density, community composition was similar in the pre- and post-project studies (Tables 13 and 14). The community was clearly dominated by chironomids in both studies - in both pools and riffles, in both fall and spring. Oligochaetes tended to be the next most abundant group. Nematodes were more abundant in the post-project than pre-project survey.

In both this and the previous study, macroinvertebrate density tended to be greater in fall than spring. Scouring flow in winter and early spring probably accounts for much of the seasonal difference (Payne and Miller 1991). Riffles showed a greater reduction of invertebrate density in spring versus fall in the previous study (Payne et al. 1991; Payne and Miller 1991). In the first study, seasonal differences were more pronounced for fully aquatic oligochaetes than for chironomids with an aerial dispersal adult stage in their life history. In the present study, there was no apparent difference in pool and riffle susceptibility to scourassociated reductions of macroinvertebrate density. 


\begin{tabular}{|c|c|c|c|c|c|c|c|}
\hline \begin{tabular}{|l|} 
Table 12 \\
Mean Dens \\
Three Chan
\end{tabular} & $\begin{array}{l}\text { Idividuals pe } \\
\text { ypes and Ty }\end{array}$ & $\begin{array}{l}\text { uare N } \\
\text { mplin }\end{array}$ & $\begin{array}{l}\text { er) of } N \\
\text { eriods }\end{array}$ & roinve & rate & Fron & les in \\
\hline & & & Fall & & & Spring & \\
\hline Order & Family & RC & $\mathrm{HC}$ & UC & RC & HC & UC \\
\hline Bivalvia & & 2067.51 & 87.76 & $=244.73$ & (20.00 & 16.88 & 499.91 \\
\hline Coleoptera & Dryopidae & 0.00 & 0.00 & 0.00 & 0.00 & 0.00 & 0.00 \\
\hline & \begin{tabular}{|l|} 
Dytiscidae \\
\end{tabular} & 0.00 & 0.00 & 0.00 & 0.00 & 0.00 & 0.00 \\
\hline & \begin{tabular}{|l} 
Elmidae \\
\end{tabular} & 59.07 & 50.63 & 774.26 & 46.41 & 6.33 & 217.00 \\
\hline & \begin{tabular}{|l|} 
Gyrinidae \\
\end{tabular} & 0.00 & 0.00 & 0.00 & 0.00 & 0.00 & 56.96 \\
\hline & Staphylinidae & 0.00 & 0.00 & 0.00 & 0.00 & 14.47 & 0.00 \\
\hline & Unknown & 0.00 & 35.44 & 0.00 & 0.00 & 0.00 & 0.00 \\
\hline Collembola & & 0.00 & 16.88 & 0.00 & 12.66 & 0.00 & 0.00 \\
\hline Diptera & Ceratapogonidae & \begin{tabular}{|l|}
599.16 \\
\end{tabular} & \begin{tabular}{|l|}
1504.22 \\
\end{tabular} & 957.81 & 1069.89 & \begin{tabular}{|l|}
685.53 \\
\end{tabular} & 597.11 \\
\hline & \begin{tabular}{|l|} 
Chironomidae \\
\end{tabular} & 35240.51 & 22135.86 & 21035.86 & 19333.91 & 5945.57 & 13815.73 \\
\hline & Empididae & \begin{tabular}{|l|}
253.16 \\
\end{tabular} & 322.36 & 426.16 & 16.88 & 46.41 & 119.53 \\
\hline & Nymphomyiidae & 0.00 & 0.00 & 0.00 & 0.00 & 0.00 & 0.00 \\
\hline & Simuliidae & 0.00 & 25.32 & 113.92 & 240.01 & 31.65 & 4028.57 \\
\hline & Tabanidae & 0.00 & 0.00 & 0.00 & 0.00 & 8.44 & 0.00 \\
\hline & Tanyderidae & 0.00 & 0.00 & 0.00 & 0.00 & 6.33 & 0.00 \\
\hline & Tipulidae & 0.00 & 59.07 & 0.00 & 0.00 & 6.33 & 0.00 \\
\hline & Unknown & 185.65 & 23.21 & 82.28 & 48.10 & 82.28 & 66.00 \\
\hline Ephemeroptera & Baetidae & 0.00 & 166.67 & 0.00 & 168.35 & 52.44 & 97.65 \\
\hline & Baetiscidae & 0.00 & 0.00 & 0.00 & 0.00 & 0.00 & 0.00 \\
\hline & Caenidae & 25.32 & 0.00 & 0.00 & 26.58 & 6.33 & 0.00 \\
\hline & Ephemeridae & 0.00 & 0.00 & 0.00 & 0.00 & 0.00 & 0.00 \\
\hline & Heptageniidae & 550.63 & 80.17 & 533.76 & 195.36 & 0.00 & 202.53 \\
\hline & Isonychiidae & 0.00 & 10.13 & 0.00 & 0.00 & 0.00 & 0.00 \\
\hline & Polmitarcyidae & 0.00 & 16.88 & 0.00 & 0.00 & 0.00 & 0.00 \\
\hline & Potamanthidae & 0.00 & 0.00 & 0.00 & 12.66 & 0.00 & 0.00 \\
\hline & \begin{tabular}{|l} 
Tricorythidae \\
\end{tabular} & 168.78 & 0.00 & 0.00 & 6.33 & 0.00 & 14.47 \\
\hline & \begin{tabular}{|l|} 
Unknown \\
\end{tabular} & 721.52 & 598.31 & 1649.79 & 802.22 & 341.41 & 433.09 \\
\hline Gastropoda & & 2244.73 & 109.70 & 455.70 & 18.99 & 0.00 & 0.00 \\
\hline Hirudinea & & 0.00 & 0.00 & 0.00 & 329.11 & 0.00 & 0.00 \\
\hline Hydracarina & & 1500.00 & 1677.22 & 6713.08 & 442.74 & 349.91 & 899.52 \\
\hline Isopoda & & 194.09 & 0.00 & 0.00 & 67.51 & 0.00 & 6.33 \\
\hline Megaloptera & Sialidae & 0.00 & 0.00 & 0.00 & 0.00 & 0.00 & 0.00 \\
\hline Nematoda & & \begin{tabular}{|l|}
10219.41 \\
\end{tabular} & 1528.27 & 4375.53 & 2001.63 & 458.05 & 2800.60 \\
\hline Nematomorpha & & \begin{tabular}{|l}
0.00 \\
\end{tabular} & 0.00 & 25.32 & 0.00 & 0.00 & 0.00 \\
\hline Odonata & Coenagrionidae & 0.00 & 0.00 & 25.32 & 0.00 & 0.00 & 0.00 \\
\hline & \begin{tabular}{|l|} 
Corduliidae \\
\end{tabular} & 0.00 & 0.00 & 0.00 & 0.00 & 0.00 & 0.00 \\
\hline & Gomphidae & 0.00 & 0.00 & 0.00 & 0.00 & 0.00 & 0.00 \\
\hline & Unknown & \begin{tabular}{|l|}
0.00 \\
\end{tabular} & 0.00 & 0.00 & 0.00 & 0.00 & 0.00 \\
\hline Oligochaeta & & \begin{tabular}{|l|}
10841.77 \\
\end{tabular} & 1122.78 & 4913.50 & 1709.55 & 1159.19 & 1755.15 \\
\hline Orthoptera & Tridactylidae & \begin{tabular}{|l|}
0.00 \\
\end{tabular} & 0.00 & 0.00 & 0.00 & 12.66 & 0.00 \\
\hline Plecoptera & Perlidae & 375.53 & 0.00 & 270.04 & 0.00 & 0.00 & 0.00 \\
\hline & \begin{tabular}{|l} 
Perlodidae \\
\end{tabular} & 6.33 & 33.76 & 29.54 & 83.12 & 6.33 & 73.24 \\
\hline & Unknown & 25.32 & 45.57 & 75.95 & 40.08 & 0.00 & 0.00 \\
\hline Trichoptera & Brachycentridae & 0.00 & 0.00 & 25.32 & 33.76 & 0.00 & 0.00 \\
\hline & Hydropsychidae & 12.66 & 435.44 & 276.37 & 16.88 & 12.66 & 113.92 \\
\hline & \begin{tabular}{|l|} 
Hydroptilidae \\
\end{tabular} & 497.89 & 97.05 & 824.89 & 16.88 & 12.66 & 45.21 \\
\hline & \begin{tabular}{|l|} 
Leptoceridae \\
\end{tabular} & 721.52 & 110.97 & 582.28 & 25.32 & 23.21 & 63.29 \\
\hline & \begin{tabular}{|l|} 
Philopotamidae \\
\end{tabular} & 455.70 & 77.22 & 2318.57 & 18.99 & 0.00 & 516.58 \\
\hline & \begin{tabular}{|l} 
Polycentropodidae \\
\end{tabular} & 101.27 & 6.33 & 0.00 & 0.00 & 0.00 & 0.00 \\
\hline & Psychomyiidae & 0.00 & 10.13 & 0.00 & 0.00 & 0.00 & 0.00 \\
\hline & Unknown & 565.40 & 510.13 & 907.17 & 130.38 & 84.09 & 450.27 \\
\hline
\end{tabular}




\begin{tabular}{|c|c|c|c|c|}
\hline \multicolumn{5}{|c|}{$\begin{array}{l}\text { Table } 13 \\
\text { Relative Abundance (Percent) of Macroinvertebrate Taxa From Pools } \\
\text { in the Recently Altered Portion of Luxapalila Creek Among Pre- and } \\
\text { Post-Alteration Sampling Periods and Two Seasons }\end{array}$} \\
\hline \multirow[b]{2}{*}{ Invertebrate Group } & \multicolumn{2}{|c|}{ Fall } & \multicolumn{2}{|c|}{$\begin{array}{c}\text { Spring } \\
\end{array}$} \\
\hline & Pre & Post & Pre & Post \\
\hline Amphipoda & 0.15 & 0.00 & 0.28 & 0.00 \\
\hline Bivalvia & 9.44 & 6.79 & 5.50 & 0.21 \\
\hline Coleoptera & 0.53 & 0.40 & 0.46 & 0.33 \\
\hline Collembola & 0.23 & 0.05 & 0.37 & 0.11 \\
\hline Diptera - Ceratopogonidae & 1.90 & 0.75 & 1.38 & 1.22 \\
\hline Diptera - Chironomidae & 47.91 & 53.58 & 60.55 & 69.13 \\
\hline Diptera - Empididae & 0.08 & 0.10 & 0.00 & 0.00 \\
\hline Diptera - Simuliidae & 0.00 & 0.00 & 0.00 & 1.04 \\
\hline Diptera - Tanyderidae & 0.08 & 0.00 & 0.00 & 0.00 \\
\hline Ephemeroptera & 0.15 & 2.52 & 0.09 & 4.95 \\
\hline Gastropoda & 0.46 & 2.64 & 0.83 & 0.12 \\
\hline Heteroptera & 0.00 & 0.00 & 0.00 & 0.00 \\
\hline Hirudinea & 0.23 & 0.17 & 0.18 & 0.00 \\
\hline Hydracarina & 3.88 & 2.70 & 1.65 & 1.13 \\
\hline Isopoda & 0.23 & 0.00 & 0.18 & 0.00 \\
\hline Nematoda & 7.31 & 14.19 & 6.15 & 12.42 \\
\hline Nemertea & 0.46 & 0.00 & 0.09 & 0.00 \\
\hline Odonata & 0.30 & 0.22 & 0.09 & 0.04 \\
\hline Oligochaeta & 23.69 & 13.65 & 20.00 & 8.14 \\
\hline Platyhelminthes & 0.99 & 0.00 & 1.01 & 0.00 \\
\hline Plecoptera & 0.08 & 0.36 & 0.37 & 0.68 \\
\hline Polychaeta & 0.08 & 0.00 & 0.00 & 0.00 \\
\hline Trichoptera & 1.83 & 1.88 & 0.83 & 0.48 \\
\hline
\end{tabular}




\begin{tabular}{|c|c|c|c|c|}
\hline \multicolumn{5}{|c|}{$\begin{array}{l}\text { Table } 14 \\
\text { Relative Abundance (Percent) of Macroinvertebrate Taxa From } \\
\text { Riffles in the Recently Altered Portion of Luxapalila Creek Among } \\
\text { Pre- and Post-Alteration Sampling Periods and Two Seasons }\end{array}$} \\
\hline \multirow[b]{2}{*}{ Invertebrate Group } & \multicolumn{2}{|c|}{ Fall } & \multicolumn{2}{|c|}{ Spring } \\
\hline & Pre & Post & Pre & Post \\
\hline Amphipoda & 0.00 & 0.00 & 0.10 & 0.00 \\
\hline Bivalvia & 6.08 & 3.07 & 5.93 & 0.00 \\
\hline Coleoptera & 0.14 & 0.09 & 0.15 & 0.17 \\
\hline Collembola & 0.31 & 0.00 & 0.10 & 0.05 \\
\hline Diptera - Ceratopogonidae & 0.08 & 0.89 & 0.00 & 3.98 \\
\hline Diptera - Chironomidae & 53.00 & 52.25 & 46.76 & 71.96 \\
\hline Diptera - Empididae & 0.02 & 0.38 & 0.00 & 0.06 \\
\hline Diptera - Simuliidae & 0.00 & 0.00 & 1.89 & 0.89 \\
\hline Diptera - Tanyderidae & 0.00 & 0.00 & 0.00 & 0.00 \\
\hline Ephemeroptera & 0.88 & 2.17 & 2.69 & 4.51 \\
\hline Gastropoda & 1.33 & 3.33 & 0.45 & 0.07 \\
\hline Heteroptera & 0.00 & 0.00 & 0.05 & 0.00 \\
\hline Hirudinea & 0.00 & 0.00 & 0.05 & 1.23 \\
\hline Hydracarina & 18.97 & 2.22 & 7.08 & 1.65 \\
\hline Isopoda & 0.00 & 0.29 & 0.00 & 0.25 \\
\hline Nematoda & 0.88 & 15.15 & 1.60 & 7.45 \\
\hline Nemertea & 0.74 & 0.00 & 0.30 & 0.00 \\
\hline Odonata & 0.05 & 0.00 & 0.05 & 0.00 \\
\hline Oligochaeta & 10.17 & 16.07 & 19.74 & 6.36 \\
\hline Platyhelminthes & 0.71 & 0.00 & 1.99 & 0.00 \\
\hline Plecoptera & 0.24 & 0.60 & 0.35 & 0.46 \\
\hline Polychaeta & 0.00 & 0.00 & 0.00 & 0.00 \\
\hline Trichoptera & 6.39 & 3.49 & 10.72 & 0.90 \\
\hline
\end{tabular}




\section{Summary}

Water and substratum characteristics are generally similar for riffles or pools whether in the downstream and recently channelized reach, the middle reach that has not been channelized, or the upper reach that was straightened and simplified in 1922. The sandy and unsorted gravelly substratum of pools and riffles, respectively, along with good water quality throughout, largely determines the macroinvertebrate community of this stream. Functionally, the macroinvertebrate community was similar throughout the stream, regardless of channel type or if samples had been from riffles or pools. A possible exception was the greater abundance of filter-feeding C. fluminea in the downstream, recently channelized reach. However, this might have had more to do with stream trophic dynamics rather than channel modifications.

Small, short-lived animals such as chironomid larvae, oligochaetes, nematodes, and the Asian clam, C. fluminea, dominated the stream macroinvertebrate community. The density of these invertebrates tended to increase in a downstream direction; this trend probably reflected the relatively oligotrophic nature of the upstream reaches of this clearwater stream. The canopy-less nature of the recently channelized downstream reach probably allows greater levels of autochthonous production of organic carbon during low flow than is possible in the relatively heavily shaded, narrower middle and upper reaches. Regardless of cause, it is clear that secondary production, reflected by macroinvertebrate density in general, and $C$. fluminea density in particular, increases moving downstream.

Overall, general limnological and trophic considerations appeared to overwhelm spatial patterns in biological organization that might otherwise have promoted differences among recently channelized, unchannelized, and historically channelized reaches. Dominance of small macroinvertebrates, most of which can rapidly recolonize disturbed areas, encouraged similarity rather than difference in community composition of the three types of stream reach. Indeed, even pools and riffles in this stream were similar. Pools were poorly formed and small, only slightly deeper than riffles, and probably subjected to considerable scour during high discharge. This was probably more true after than before project construction in the recently channelized reach. Gravel in this sand-channel stream was mostly limited to riffles, poorly sorted (Payne et al. 1991), and probably unstable during very high flows.

Almost certainly, relatively long-lived and sedentary animals, such as native unionid mussels, are more likely to thrive in the unchannelized than recently 
channelized reaches of the stream, and even in that reach truly stable shoals of sand and gravel barely exist. Compared with $C$. fluminea, most native unionids require long-term stability of sand or gravel substratum to establish moderately dense assemblages (Payne et al. 1989). Long-term monitoring will be necessary to evaluate the status of the sparse unionid fauna in the unchannelized middle reach of the river.

The ability of the Luxapalila Creek macroinvertebrate fauna to recolonize disturbed areas was evident from the pre- and post-project comparisons of the recently channelized lower reach of the stream. Short-lived and relatively small chironomids, oligochaetes, nematodes, and $C$. fluminea, that in combination comprise the very large majority of the macroinvertebrate fauna, fully recolonized the recently channelized lower reach. Both in that reach in Columbus, MS, and in the historically channelized but not recently disturbed reach in Alabama, basic community composition was the same as in 1987-1989. In addition, total density of macroinvertebrates was higher in the present study. There was no evidence of the macroinvertebrate community of Luxapalila Creek having been detrimentally affected by flood control measures. Indeed, production of dominant organisms probably increased. 


\section{References}

Barbour, M. T., Gerritsen, J., Snyder, B. D., and Stribling, J. B. (1999). Rapid bioassessment protocols for use in streams and wadeable rivers: Periphyton, benthic macroinvertebrates and fish. $2^{\text {nd }}$ ed., EPA 841-B-99-002, U.S. Environmental Protection Agency, Office of Water, Washington, DC.

Jandel Corporation. (1995). "SigmaStat for Windows version 2.0 statistical software," San Rafael, CA.

Miller, A. C., and Bingham, C. R. (1987). "A hand-held benthic core sampler," Journal of Freshwater Ecology 4: 77-81.

Payne, B. S., and Miller, A. C. (1991). "The structure of dominant invertebrate assemblages in a small southeastern stream," Journal of Freshwater Ecology 6: 257-266.

Payne, B. S., Miller, A. C., Hartfield, P. D., and McMahon, R. F. (1989). "Variation in size demography of lotic populations of Corbicula fluminea (Muller)," The Nautilus 103: 78-82.

Payne, B. S., Miller, A. C., Miller-Way, C., and Bingham, C. R. (1991). "Macroinvertebrates of Luxapalila Creek, Mississippi and Alabama, 1987-89," Miscellaneous Paper EL-91-5, U.S. Army Engineer Waterways Experiment Station, Vicksburg, MS.

Standford, J. A., and Ward. J. V. (1979). "Stream regulation in North America." The ecology of regulated streams. J. V. Ward and J. A. Stanford, ed., Plenum Press, New York, 215-236.

Zar, J. H. (1987). Biostatistical analysis. $2^{\text {nd }}$ ed., Prentice Hall, Englewood Cliffs, NJ. 


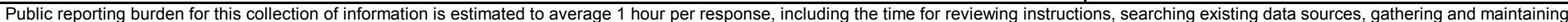

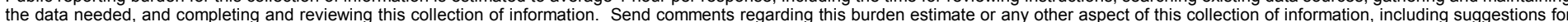

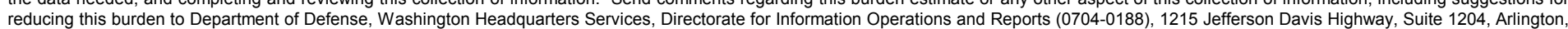

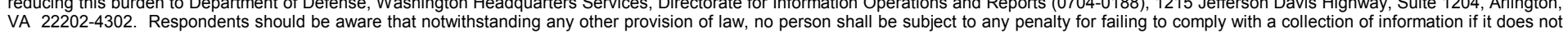
display a currently valid OMB control number. PLEASE DO NOT RETURN YOUR FORM TO THE ABOVE ADDRESS.

\begin{tabular}{l|c} 
1. REPORT DATE (MMMM-YYYY) & $\begin{array}{c}\text { 2. REPORT TYPE } \\
\text { Feptember } 2001\end{array}$ \\
\hline
\end{tabular}

\section{TITLE AND SUBTITLE}

Macroinvertebrate Communities and Habitat in Luxapalila Creek, Mississippi and Alabama

3. DATES COVERED (From - To)

5a. CONTRACT NUMBER

5b. GRANT NUMBER

5c. PROGRAM ELEMENT NUMBER

5d. PROJECT NUMBER

5e. TASK NUMBER

5f. WORK UNIT NUMBER

8. PERFORMING ORGANIZATION REPORT NUMBER

U.S. Army Engineer Research and Development Center

Environmental Laboratory, 3909 Halls Ferry Road

Vicksburg, MS 39180-6199;

Mississippi State University

Mississippi State, MS 39762

9. SPONSORING / MONITORING AGENCY NAME(S) AND ADDRESS(ES)

U.S. Army Engineer District, Mobile

Mobile, AL 36628-0001
ERDC/EL TR-01-34

10. SPONSOR/MONITOR'S ACRONYM(S)

11. SPONSOR/MONITOR'S REPORT NUMBER(S)

\section{DISTRIBUTION / AVAILABILITY STATEMENT}

Approved for public release; distribution is unlimited.

\section{SUPPLEMENTARY NOTES}

\section{ABSTRACT}

A survey of macroinvertebrates and their habitat was conducted in Luxapalila Creek soon after completion of flood control measures in the lower reach near Columbus, MS, 1998-2000. The results of this study were compared with a similar preconstruction survey conducted in 1987-1989. There was no evidence of adverse effects of construction on the benthic macroinvertebrate community. Recently channelized, unchannelized, and historically channelized portions of the stream differed somewhat in geomorphological (e.g., stream width) and other physical habitat characteristics (e.g., canopy coverage). However, at all sites macroinvertebrate community composition was marked by similarity, both functionally and structurally. Substratum and water quality conditions were also similar throughout the stream. Density of macroinvertebrates was greatest in the recently channelized downstream reach. Collector-filterers (mostly the Asian clam, Corbicula fluminea) were least abundant in the upstream reach that was channelized 80 years ago. It is likely that these patterns reflect quantitative trophic difference along an upstream to downstream gradient usual for streams of this size. Short-lived, small invertebrates such as midges and worms (chironomids, oligochaetes, and nematodes) numerically dominated the fauna. Drift of the worms and aquatic stages of midges, combined with aerial dispersal of adult midges, contributes to the rapid colonization ability and uniformity of community composition of the macroinvertebrate community that characterizes Luxapalila Creek.

\section{SUBJECT TERMS}

Channelization

Macroinvertebrates

Luxapalila Creek Mississippi

16. SECURITY CLASSIFICATION OF:

17. LIMITATION

\section{a. REPORT}

UNCLASSIFIED

b. ABSTRACT
UNCLASSIFIED

c. THIS PAGE

UNCLASSIFIED
17. LIMITATION OF ABSTRACT

\begin{tabular}{|l|} 
18. NUMBER \\
OF PAGES \\
\cline { 2 - 2 } \\
\cline { 2 - 2 }
\end{tabular}

19a. NAME OF RESPONSIBLE PERSON

19b. TELEPHONE NUMBER (include area code) 